PERIS, J.B. y G. STÜBING -1985-Silene oropediorum, una especie nueva para la flora europea. Anales Jard. Bot. Madrid 41(2): 453. RIVAS-MARTÍNEZ, S., M. COSTA, S. CASTROVIEJO y E. VALDÉS -1980- Vegetación de Doñana (Huelva, España). Lazaroa 2: 5-190.

RIVAS-MARTÍNEZ, S., M.E. GARCÍA y A. PENAS -1991- Revisión taxonómica de la sección Aizopsis DC. del género Draba L. en la Península Ibérica. Candollea 46: 439-473.

SCHULZ, O.E. -1957- Cruciferae- Sisymbrieae. In: Engler, A. (Ed.), Das Pflanzenreich, 86. Neudruck.

SAGREDO, R. -1987- Flora de Almería. Inst. Est. Almerienses. Almería.

TALAVERA, S. -1990- Silene L. In: Castroviejo, S. et al. (Eds.). Flora Ibérica. Vol. II. Real Jardín Botánico de Madrid. CSIC.

UBERA, J.L. y B. VALDÉS - 1983- Revisión del género Nepeta (Labiatae) en la Península Ibérica e Islas Baleares. Lagascalia 12(1): 3-80.
VALDÉS, B., S. TALAVERA y E. FERNÁNDEZGALIANO - 1987- Flora vascular de Andalucía occidental. Vol II. Ketres editora. Barcelona. VALLE, F. y C. DÍAZ DE LA GUARDIA - 1987- La Alfaguara y su entorno vegetal. Granada.

VOGT, R. -1991- Die Gattung Leucanthemum Mill. (Compositae-Anthemidae) auf der Iberischen Halbinsel. Monografías del Real Jardín Botánico 10. CSIC.

Aceptado para su publicación en Junio de 1998

Dirección de los autores. A. Lora González: Dpto. de Ciencias y Recursos Agrícolas y Forestales, UCO. Jardín Botánico de Córdoba. Avda. de Linneo s/n. 14004 Córdoba. J.L. Vivero Pol: Jardín Botánico de Córdoba. Avda. de Linneo s/n. 14004 Córdoba.

\title{
58. HYPOCHAERIS ANGUSTIFOLIA (ASTERACEAE): DISTRIBUCIÓN, ECOLOGÍA Y FITOSOCIOLOGÍA
}

\author{
Antonio GALÁN DE MERA y José Alfredo VICENTE ORELLANA
}

Hypochaeris angustifolia (Asteraceae): distribution, ecology and phytosociology.

Palabras clave. Hypochaeris, fitogeografía, Marruecos.

Key Words. Hypochaeris, phytogeography, Morocco.

El género Hypochaeris L. está Mediterránea y América del Sur (Cerbah et al., ámpliamente repartido en el mundo con dos 1995). De todas sus especies, tal vez la menos grandes centros de dispersión, la cuenca conocida es $H$. angustifolia (Litard. \& Maire) Maire, puesto que la mayor parte del material

Este trabajo ha sido realizado con los fondos del proyecto de investigación 12/95 de la Universidad San Pablo-CEU. 


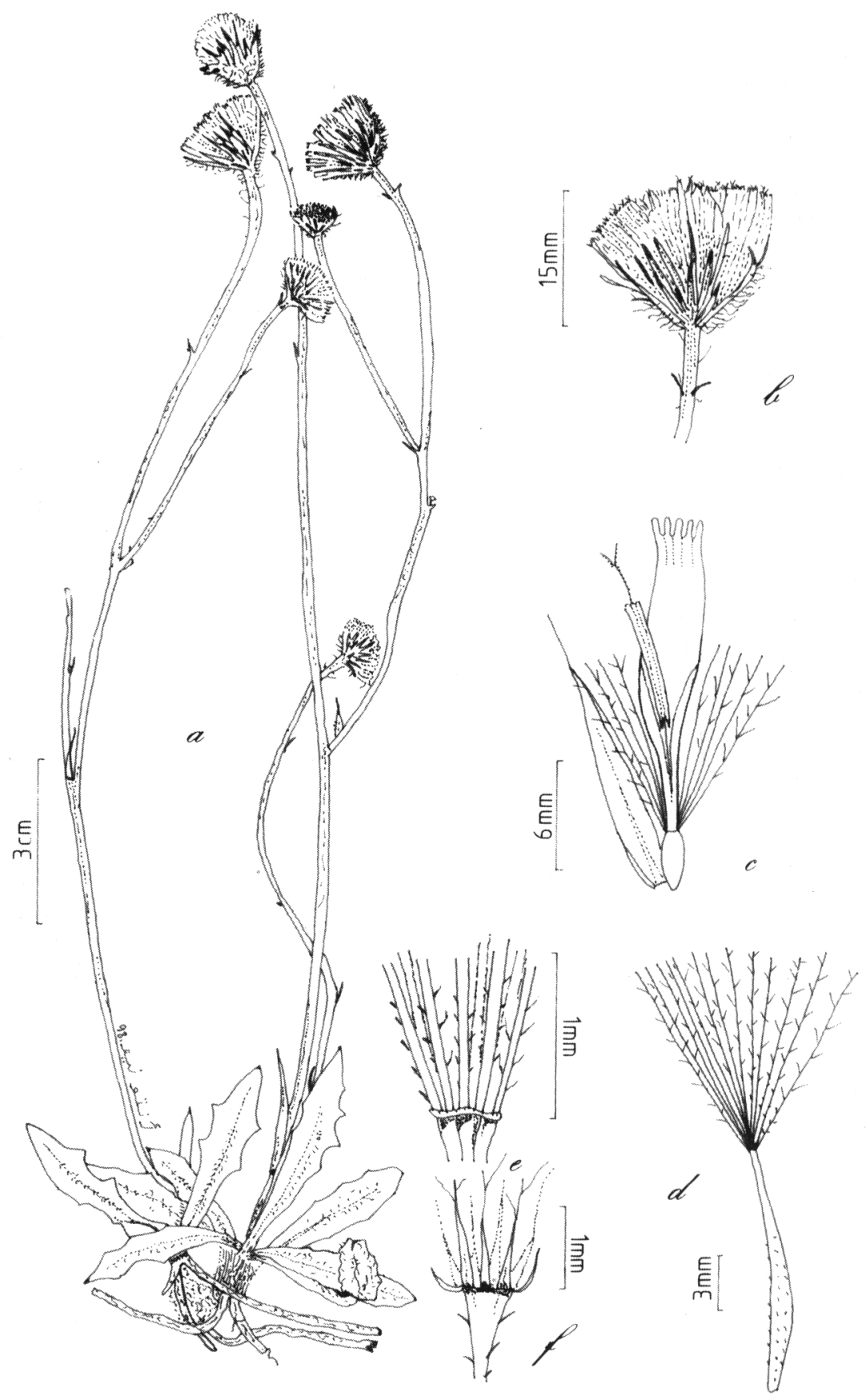

Figura 1. Hypochaeris angustifolia (USP 198698): a) hábito; b) detalle del capítulo; c) flor y bráctea del capítulo; d) aquenio con su vilano; e) detalle de la base del vilano; f) detalle de la base del vilano de $H$. aggr. laevigata (USP 198198). a) habit; b) detail of the head; c) flower and floral leave; d) akene with it pappus; e) detail of the base of the pappus; $f$ ) detail of the base of H. aggr. laevigata pappus (USP 198198). 
disponible en los herbarios procede de finales del siglo XIX y principios del XX. Esta planta fue descrita por Litardière y Maire (1924) como H. laevigata (L.) Ces., Pass. \& Gibelli subsp. angustifolia Litard. \& Maire, lo que conlleva su pertenencia a la sección Seriola L. - plantas con los vilanos, al menos, biseriados, los pelos más anchos en la base y los radios externos setiformes (Boissier, 1875; Fiori y Paoletti, 1903). Sin embargo, H. angustifolia (fig. 1) es una planta bien diferente a las del grupo laevigata (Galán de Mera y Vicente Orellana, 1998), ya que el vilano es uniseriado y constituído por cerdas de anchura constante en toda su longitud (sección Achyrophorus Adans.).

\section{Material de herbario y distribución}

MARRUECOS. HAUT ATLAS: Âï-Hani, 2000 m, R. Maire, 26-VI-1939, MPU; Âit Rich, VI-1926, Humbert, MPU; Glaoua, Jerekten, 1200 m, R. Maire, 21-VII-1926, MPU; Glaoua, Telouet, 1980 m, R. Maire, 8-VII-1924, MPU; Goundafa, $H^{\text {tc }}$ vallée de l'acif Agoudim, pelouses à Eryngium variifolium entre les bergeries et le village d'Âï Ioub, $2750 \mathrm{~m}, R$. de Litardière, 3-VIII-1923, MPU (material tipo); Qal'at Mgouna, R. Maire, 21-VI-1939, MPU; Ourika, 1000 m, R. Maire, 8-VII-1921; Ibídem, lieux humides, sources et suintements sur les argiles et grès rouges, près Anfegeïn, 2100 m, R. Maire, 9-VII-1921, MPU ; Tislit,

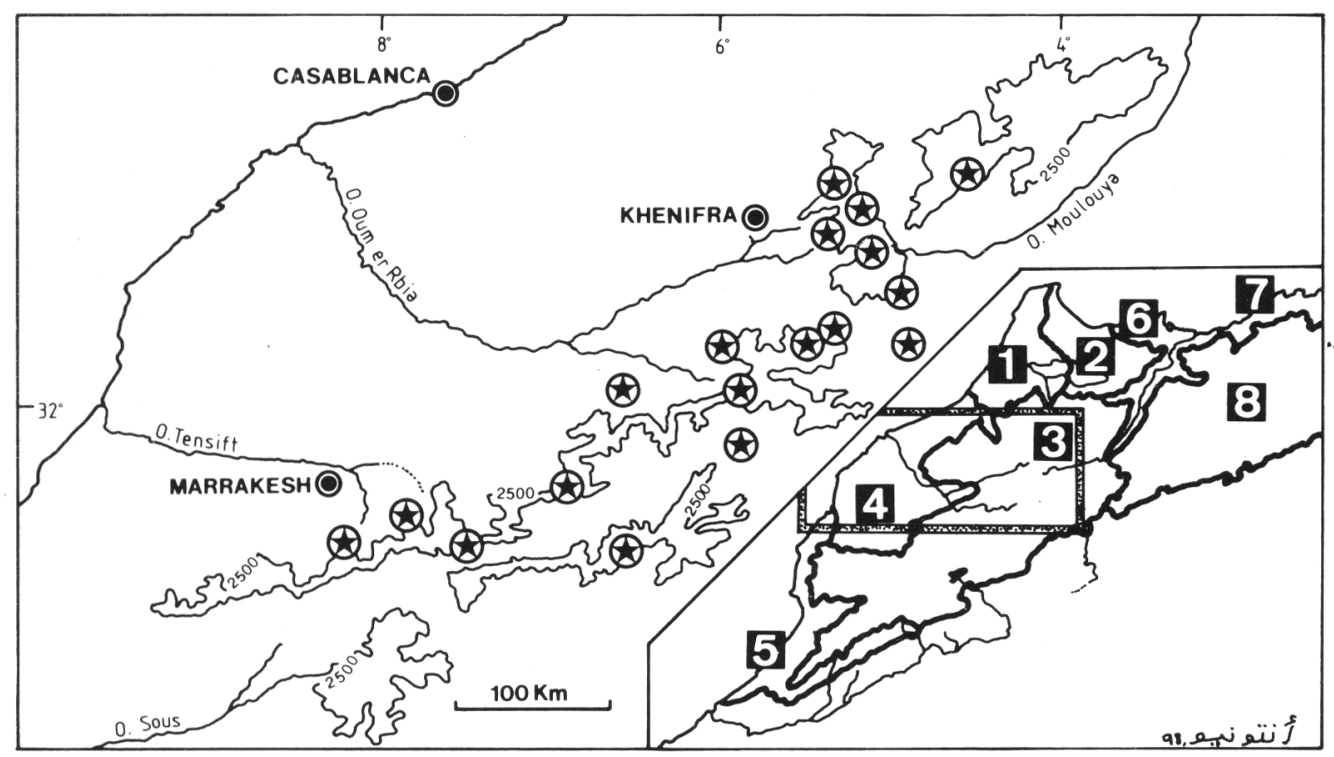

Figura 2. Distribución de Hypochaeris angustifolia en la cordillera del Atlas (Marruecos). En el recuadro de la derecha se indica la división fitogeográfica de la parte extrasahariana de Marruecos y oeste de Argelia (tomado de Deil y Galán de Mera, 1998): superprovincia Iberomarroquí-atlántica: 1- provincia TingitanoOnubo-Algarviense, 2- provincia Rifeña, 3- provincia Atlásica, 4-provincia Atlántica, 5- provincia Suroccidental; superprovincia Mauritánica-mediterránea: 6- provincia Muluyense, 7- provincia OranoKabiliense, 8- provincia Hauts Plateaux. Distribution of H. angustifolia in the Atlas mountains. The window of the right is the phytogeographical division of the extra-Saharien part of Morocco and western Algeria (from Deil \& Galán de Mera, 1998): Iberomarroqui-atlantica superprovince: 1- Tingitano-OnuboAlgarviense province, 2-Rifeña province, 3-Atlasica province, 4-Atlantica province, 5-Sur-occidental province; Mauritanica-mediterranea superprovince: 6 -Muluyense province, 7-Orano-Kabiliense province, 8- Hauts Plateaux province. 


\begin{tabular}{|c|c|c|c|c|c|c|c|c|c|c|}
\hline Número de orden & 1 & 2 & 3 & 4 & 5 & 6 & 7 & 8 & 9 & 10 \\
\hline Area $\mathrm{m}^{2}$ & 100 & 100 & 100 & 100 & 50 & 100 & 50 & 50 & 50 & 30 \\
\hline Altitud (m) & 1735 & 1735 & 1735 & 1760 & 1745 & 1760 & 1745 & 2100 & 1990 & 2100 \\
\hline Número de especies & 24 & 23 & 18 & 17 & 16 & 16 & 13 & 25 & 24 & 19 \\
\hline
\end{tabular}

Características de Phleo-Poetum bulbosae

Phleum bertolonii

Diferenciales de trifolietosum repentis

Trifolium repens

Hypochaeris angustifolia

Festuca arundinacea

Trifolium dubium

Phleum pratense

Poa pratensis

Características de Potentillo-Campanulion

Campanula filicaulis

Potentilla maura

Plantago coronopus subsp. cupani

Carduncellus pinnatus

Carduncellus rhaponticoides

Trifolium humile

Características de Poetalia y Poetea

Poa bulbosa

Trifolium subterraneum

Ranunculus millefoliatus

Paronychia argentea

Cerastium glomeratum

\begin{tabular}{|c|c|c|c|c|c|c|c|c|}
\hline+ & 1 & & 1 & 1 & 1 & 1 & 2 & 2 \\
\hline 2 & 2 & 2 & 2 & . & 1 & . & 1 & 1 \\
\hline 1 & 1 & 3 & 1 & . &. & . & 2 & . \\
\hline+ & . & 1 & 1 & . & . & . & . & + \\
\hline+ & 1 & . & . & . & 1 & . & . & \\
\hline . & + & . & . & . & & . & . & \\
\hline 3 & 4 & 4 & 4 & 3 & 3 & 4 & 2 & 3 \\
\hline 2 & 2 & 2 &. & . & . & $\cdot$ & & 1 \\
\hline+ & 1 & . & . & . & . & . & 1 & 1 \\
\hline+ & . & . & 1 & $\cdot$ & 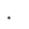 & • & & . \\
\hline 1 & 1 & . & . & . & 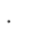 & . & 1 & 1 \\
\hline
\end{tabular}

Características de Molinio-Arrhenatheretea

Carex divisa

Festuca rubra

Hieracium pseudopilosella

Trisetaria flavescens

Dactylis glomerata

Lotus corniculatus var. pilosus

Anthoxanthum ovatum

Arrhenatherum elatius subsp. bulbosum

Cynodon dactylon

Taraxacum atlanticum

Compañeras

Evax pygmaea

Herniaria glabra

Convolvulus lineatus

Sagina apetala

Eryngium bourgatii

Myosotis stricta

Armeria choulettiana

Rumex angiocarpus

Juncus bufonius

Capsella bursa-pastoris

Alyssum granatense

Hordeum murinum

Catananche caespitosa

Bromus hordeaceus

Cynara histrix

Bupleurum spinosum

Sedum caespitosum

Erophila verna

\begin{tabular}{|c|c|c|c|c|c|c|c|c|}
\hline 4 & 3 & 2 & 3 & 2 & & 2 & & + \\
\hline 1 & . & 1 & 1 & . & 1 & 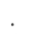 & 4 & 4 \\
\hline & . & . & 2 & & 2 & 1 & 3 & 2 \\
\hline 1 & . & . & 1 & 1 & 2 & 1 & . & 1 \\
\hline . & + & 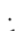 & . & + & . & 1 & & 1 \\
\hline . & 1 & 1 & . & & . & . & 1 & 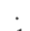 \\
\hline . & $\cdot$ & $\cdot$ & . & 1 & 1 & . & . & 1 \\
\hline & $\cdot$ & $\cdot$ & & 1 & & $\cdot$ & . & \\
\hline - & $\cdot$ & $\cdot$ & . & $\cdot$ & 1 & . & 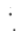 & 1 \\
\hline . & . & . & . & . & . & . & + & . \\
\hline
\end{tabular}

Otros táxones: Hypochaeris glabra + en 1; Aphanes maroccana 1, Scleranthus annuus subsp. polycarpos 1 en 2; Cerastium gibraltaricum 1, Eryngium triquetrum + , Caucalis caerulescens + en 3; Galium corudifolium + en 4; Echinaria capitata 1, Helianthemum croceum +, Thymus serpyllum subsp. atlanticus + en 5; Anthemis pedunculata +, Erodium cicutarium 1 en 6 ; Minuartia campestris + , Ranunculus muricatus + , Medicago minima,+ Ononis thomsonii 1 en 8.Localidades.- 1-7: Valle de Bekrite, Khénifra. 8-10: Col du Zad, entre Timahdite y Midelt.

Tabla 1. Phleo nodosi-Poetum bulbosae trifolietosum repentis subass. nova (Potentillo-Campanulion, Poetalia, Poetea) 
2300 m, R. Maire, 27-VI-1939; MOYEN ATLAS: Âit Makhlouf, 1400 m, R. Maire, 18VIII-1924, MPU; Beni-Mellal, Tassemit, 32/ $18 \mathrm{~N}-6 / 15 \mathrm{~W}$, rochers calcaires, 1850-1900 m, A. Charpin, F. Jacquemoud \& D. Jeanmonod, 6-VI-1980, G. Bekrite, 1900 m, R. Maire, 5VIII-1924, MPU; Ibídem, Aghbalo Immouyer, 1900 m, R. Maire, 4-VIII-1924, MPU; Bou Iblane, Neskedal, $2000 \mathrm{~m}, R$. Maire, 28-VI1927, MPU; Col du Zad, entre Timahdite y Midelt, $2100 \mathrm{~m}$, A. Galán de Mera y J. A. Vicente Orellana, 25-VI-1996, USP 198698, 198798, 198898; Ksar es-Souk, R. Maire, 2-V1927, MPU; Masker, 2500 m, R. Maire, 6-VII1934, MPU; Midelt, 1500 m, R. Maire, 29-VI1939; Ibídem, l'Oued Outat, R. Maire, 10-VII1919; Seguina, $1600 \mathrm{~m}, R$. Maire, 11-VIII1924, G, MPU; Senoual, 2000 m, R. Maire, 28VI-1938, MPU; Tizi, Ishi, 1650 m, R. Maire, 21-VI-1936, MPU.

Hypochaeris angustifolia es un endemismo de la cordillera del Atlas (fig. 2) que caracteriza a la provincia fitogeográfica Atlásica (Deil y Galán de Mera, 1998) ya que se extiende desde el macizo de Bou Iblane (Moyen Atlas) hasta el Toubkal (Haut Atlas).

\section{Ecología y fitosociología}

Si bien Hypochaeris aggr. laevigata $(H$. laevigata y $H$. leontodontoides Ball) reune a plantas fisurícolas, las especies de la sección Achyrophorus, tanto euro-mediterráneas como americanas, forman parte de pastizales (Cabrera, 1963; Pignatti, 1982). De Europa, $H$. maculata L. está sobre todo en las comunidades basófilas de Festucetalia valesiacae Br.-B1. \& R. Tx. 1943, y H. uniflora Vill. es característica de Nardion Br.-Bl. in Br.-Bl. \& Jenny 1926 (Oberdorfer, 1990). Según Quézel (1957), H. angustifolia se trata de una característica de la alianza de megaforbios Eryngion variifoliae Quézel 1957, aunque a juzgar por la tabla que ofrece este autor bien podría tratarse de una característica de los pastizales de suelos húmedos de Molinio-Arrhenatheretea R. Tx. 1937. En las laderas que rodean al Col du Zad, entre Timahdite y Midelt (Moyen Atlas), hemos levantado inventarios fitosociológicos con $H$. angustifolia, donde se asocia a otras plantas de Molinio-Arrhenatheretea formando mosaico con los pastizales de la asociación Phleo nodosi-Poetum bulbosae Benabid 1988 (Potentillo maurae-Campanulion filicaulis Galán de Mera \& Vicente Orellana 1997. Poetalia bulbosae Rivas Goday \& RivasMartínez 1963, Poetea bulbosae Rivas Goday \& Rivas-Martínez 1978). Su significado ecológico es el tránsito de las comunidades de Poetea hacia Molinio-Arrhenatheretea en los suelos básicos más húmedos próximos a arroyadas o en concavidades donde la humedad permanece más tiempo. Esta pequeña diferencia edáfica permite describir a la nueva subasociación trifolietosum repentis (tabla 1 , typus: invent. 8) donde, aparte de Trifolium repens $\mathrm{L}$., podemos destacar diferenciales como Festuca arundinacea Schreber, H. angustifolia, Phleum pratense L., Poa pratensis L. y Trifolium dubium Sibth.

AGRADECIMIENTOS. Gracias a los conservadores de los herbarios BC, BM, E, FB, G, GE, MA, MAF, MO, MPU, NEU, OXF, P y UBT que pusieron a nuestra disposición su material de Hypochaeris.

\section{BIBLIOGRAFÍA}

BOISSIER, E. -1875- Flora orientalis III. Genève. CABRERA, A.L. -1963- Estudios sobre el género Hypochoeris. Bol. Soc. Argent. Bot. 10 (2-3): 166-195.

CERBAH, M., J. COULAUD, B. GODELLE y S. SILJAK-YAKOVLEV -1995- Genome size, fluorochrome banding, and karyotype evolution in some Hypochoeris species. Genome 38: 689695.

DEIL, U. y A. GALÁN DE MERA -1998Contribution à la connaissance de la 
phytosociologique et biogéographie des groupements rupicoles calcaires du Maroc. Bull. Inst. Bot. Rabat (en prensa).

FIORI, A. y G. PAOLETTI -1904- Flora analitica d'Italia III. Padova.

GALÁN DE MERA, A. y J.A. VICENTE ORELLANA -1998- Lectotypification of Hypochaeris leontodontoides and other notes on the Hypochaeris laevigata group (Asteraceae). Taxon 47: 115-116.

LITARDIÈRE, R. y R. MAIRE-1924-Contribution à l'étude de la flore du Grand Atlas. Mém. Soc. Sci. Nat. Maroc 4: 1-32.
OBERDORFER, E. -1990- Pflanzensoziologische Excursionsflora. Stuttgart.

PIGNATTI, S. -1982- Flora d'Italia III. Bologna.

QUÉZEL, P. -1957-Peuplement végétal des hautes montagnes de l'Afrique du Nord. Paris.

Aceptado para su publicación en Junio de 1998

Dirección de los autores: Laboratorio de Botánica. Departamento de Biología. Universidad San PabloCEU. Ap. 67. E- 28660 Boadilla del Monte (Madrid). Fax: 91351049 6, E-mail: agalmer@ceu.es

\title{
59. ACERCA DE TRES ESPECIES NITRÓFILAS EN LA PROVINCIA DE ALMERÍA
}

\author{
Elías DANA, Juan F. MOTA, Javier CABELLO y Julio PEÑAS
}

Concerning three nitrofilous species in Almeria province

Palabras clave. malas hierbas, corología, Almería, España.

Key words. weeds, chorology, Almería, Spain.

Con motivo de un trabajo de investigación sobre vegetación urbana en Almería, hemos registrado la presencia de más de doscientos taxones nitrófilos. De este contingente, seleccionamos tres especies para la presente nota: Spergula fallax, Chamaesyce serpens y Atriplex semibaccata, por su particular interés biogeográfico y malherbológico. De todas ellas, sólo la primera puede considerarse autóctona. Bajo el nombre de cada taxón indicamos las coordenadas UTM de la zona de herborización y la referencia de herbario.
Spergula fallax (Lowe) E. H. L. Krause.

ALMERÍA: Almería ciudad, UTM 30SWF4979, $10 \mathrm{~m}$., cultivos abandonados alrededor de la ciudad, 15/03/97, E. Dana, Herbario de la Universidad de Almería 585.

Terófito disyunto asiático-mediterráneo occidental (incluyendo Canarias y Madeira), con desarrollo invernal y primaveral. Su presencia en el continente europeo sólo es conocida con certeza en Almería, concretamente en dos puntos distantes entre sí unos $60 \mathrm{Km}$, los Campos de Dalías y la comarca 